\title{
CHARACTERISTIC CLASSES FOR THE DEFORMATION OF FLAT CONNECTIONS ${ }^{1}$
}

\author{
BY
}

\section{HUEI-SHYONG LUE}

\begin{abstract}
In this paper, we study the secondary characteristic classes derived from flat connections. Let $M$ be a differential manifold with flat connection $\omega_{0}$. If $f$ is a diffeomorphism of $M$, then $\omega_{1}=f^{*} \omega_{0}$ is another flat connection. Denote by $\alpha$ the difference of these two connections. Then $\alpha$ and its exterior covariant derivative $D \alpha$ are both tensorial forms on $M$. To each invariant polynomial $\varphi$ of $\mathrm{GL}(n, \mathrm{R})$, where $n=\operatorname{dim} M, \varphi(\alpha ; D \alpha)$ is a globally defined form on $M$. The class $\{\varphi(\alpha ; D \alpha)\} \in H(M ; R)$ for $\operatorname{deg} \varphi>1$ gives rise to an obstruction of the deformability from $\omega_{0}$ to $\omega_{1}$. In particular, we prove that $(+)$ and $(-)$ connections, in the sense of $E$. Cartan, cannot be deformed to each other.
\end{abstract}

Introduction. Only very recently has the theory of secondary characteristic classes been extensively studied. While the primary characteristic classes deal with the topological features of a manifold, the secondary characteristic classes are primarily concerned with its underlying geometric structures.

Let $P$ be a principal $G$-bundle over $M$ with a connection form $\omega$ on $P$. We denote by $\Omega$ the curvature form of $\omega$. Let $\varphi$ be an invariant symmetric polynomial of degree $k$ on the Lie algebra $g$ of $G$. If we put

$$
\Omega_{t}=t d \omega+\left(t^{2} / 2\right)[\omega, \omega] \text { for } t \in[0,1],
$$

and

$$
T \varphi(\omega)=k \int_{0}^{1} \varphi\left(\omega ; \Omega_{t}\right) d t
$$

then we have $d T \varphi(\omega)=\varphi(\Omega)$.

The form $\varphi(\Omega)$ can be projected to the base manifold $M$ and it is a closed form. It is well known that the class $\{\varphi(\Omega)\} \in H^{2 k}(M ; R)$ is independent of the choice of the connection form $\omega$ and is called the characteristic class of $P$ defined by $\varphi$.

Contrary to these facts about characteristic classes, the form $T \varphi(\omega)$ is in general defined on the bundle space $P$ and depends on the connection. In [3],

Received by the editors November 7, 1974 and, in revised form, March 21, 1975.

AMS (MOS) subject classifications (1970). Primary 53A15, 55F40, $53 \mathrm{C05}$.

${ }^{1}$ )This paper is part of the author's doctoral dissertation written under the direction of Professor T. Nagano at the University of Notre Dame. The author wishes to express his hearty thanks to Professor Nagano for his encouragement and valuable suggestions. 
Chern and Simons gave a formula (see Proposition (1.4) and a simple proof will be given in \$4) which described how this form depends on the connection. Things become more interesting when the characteristic form $\varphi(\Omega)$ vanishes. In this case, $T \varphi(\omega)$ is a closed form and it represents an element $\{T \varphi(\omega)\}$ of the deRham cohomology group of $P$. There are many interesting cases where the characteristic form $\varphi(\Omega)$ vanishes.

Let $F$ be a foliation of codimension $q$ on a manifold $M$. If we denote by $N=T M / F$ the normal bundle of $F$ in $M$, then Bott's vanishing theorem says that, with respect to any basic connection $\omega$ of the normal bundle $N, \varphi_{j}(\Omega)=0$ for $j \geqslant[q / 2]+1$, where $\varphi_{j}$ is the $j$ th Pontrjagin polynomial on $\mathrm{gl}(q ; R)$. The secondary characteristic classes derived in this way are called the generalized Godbillon-Vey classes which have been studied extensively by Bott, Haefliger, Kamber and Tondeur.

In [3], Chern and Simons considered a manifold immersed in Euclidean space and obtained some necessary conditions for the immersion to be conformal. Kobayashi and Ochiai [6] gave a unified treatment of the Chern-Simons' results when $M$ has a $G$-structure of order two.

In [7], [8], Kamber and Tondeur constructed certain characteristic invariants $\Delta_{*}\left(x_{i}\right) \in H^{2 i-1}(M ; R)$ for $i=1,3,5, \ldots$ associated to each affine manifold $M$. (See Theorem 1.) They are deformation invariants for $i>1$.

In this paper, we shall investigate more closely the secondary characteristic classes which are derived from flat connections. If $\omega_{0}$ is a flat connection on $M$, and if $f$ is a diffeomorphism of $M$, then $f^{*} \omega_{0}=\omega_{1}$ gives another flat connection. Let $\alpha$ denote the difference of these two connections. Then $\alpha$ is a tensor field of type $(1,1)$ on $M$. The exterior covariant derivative $D \alpha$ of $\alpha$ with respect to the connection $\omega_{0}$ is another tensor field. (For definition of $D \alpha$, see $\S 1$.) Hence, to each invariant symmetric polynomial $\varphi$ on $g l(n ; R), n=\operatorname{dim} M, \varphi(\alpha ; D \alpha)$ is a globally defined form on $M$. It is a closed form. Such a class $\{\varphi(\alpha ; D \alpha)\} \in$ $H(M ; R)$ gives an obstruction to the deformability of $\omega_{0}$ to $\omega_{1}$ (Theorem 2). Using this fact, we can prove that the $(+)$ and $(-)$ connections on $S O(3)$ cannot be deformed to each other via flat connections.

Following Bott's idea in [1], we give a systematic treatment of transgression forms in $\S 4$. This treatment greatly simplifies the proofs of Chern-Simons' formula (Proposition (1.4)) and Heitsch's formula (Corollary (2.2)). On the other hand, this treatment gives us an invariant in each connected component of the space of flat connections.

1. Generalities. In this section, we will fix the notations and derive the basic formulas which will be used in the subsequent sections. Mainly, we will follow the notations in [5]. 
If $M$ is a differentiable manifold and $V$ a finite-dimensional vector space, then we denote by $\Lambda^{q}(M ; V)$ the space of all $V$-valued $q$-forms on $M$. If $V=R$, we put $\Lambda^{q}(M)=\Lambda^{q}(M ; R) . \Lambda^{q}(M ; V)$ is a module over $\Lambda^{0}(M)$ and the direct sum of $\Lambda^{q}(M ; V)$ is denoted by $\Lambda(M ; V)$

$$
\Lambda(M ; V)=\sum_{q} \Lambda^{q}(M ; V) .
$$

Let $G$ be a Lie group with its Lie algebra denoted by $\boldsymbol{g}$. If $\left\{e_{i}\right\}_{i=1}^{n}$ is a basis of $g$ with structure constants $\left\{c_{j k}^{i}\right\}$, that is, $\left[e_{j}, e_{k}\right]=c_{j k}^{i} e_{i}$, then we have the induced map denoted by the same symbol $[]:, \Lambda^{q}(M ; g) \times \Lambda^{r}(M ; g) \rightarrow$ $\Lambda^{q+r}(M ; g)$ which is expressed as follows:

For $\xi=\Sigma_{i=1}^{n} \xi^{i} \otimes e_{i}, \eta=\Sigma_{j=1}^{n} \eta^{j} \otimes e_{j}$,

$$
[\xi, \eta]=c_{j k}^{i} \xi^{j} \wedge \eta^{k} \otimes e_{i} \text {. }
$$

It is easy to verify the following formulas:

$$
\begin{aligned}
& {[\xi, \eta]=(-1)^{q r+1}[\eta, \xi] \quad \text { for } \xi \in \Lambda^{q}(M ; g), \eta \in \Lambda^{r}(M ; g),} \\
& d[\xi, \eta]=[d \xi, \eta]+(-1)^{q}[\xi, d \eta], \\
& (-1)^{q s}[\xi,[\eta, \theta]]+(-1)^{r q}[\eta,[\theta, \xi]]+(-1)^{s r}[\theta,[\xi, \eta]]=0 \\
& \text { for } \theta \in \Lambda^{s}(M ; g) .
\end{aligned}
$$

(1.3) is called the Jacobi identity. In particular, we have

$$
[\xi,[\xi, \xi]]=0 \text { for } \xi \in \Lambda^{q}(M ; g) \text {. }
$$

Let $P$ be a principal bundle over $M$ with structure group $G$. A connection form $\omega$ of $P$ is a $g$-valued 1 -form on $P$ such that

(i) $\omega(A)=A$ for $A \in \mathrm{g}$, and

(ii) $R_{g}^{*} \omega=\operatorname{Ad}\left(g^{-1}\right) \omega$ for $g \in G$.

The curvature form $\Omega$ of $\omega$ is given by $\Omega=d \omega+1 / 2[\omega, \omega]$.

Definition (1.1). Let $\rho: G \rightarrow \operatorname{GL}(V)$ be a representation. $\xi \in \Lambda^{q}(P ; V)$ is called a tensorial $q$-form on $P$ of type $(V, \rho)$ if

(i) $i(X) \xi=0$ for $X \in g$, where $i(X)$ is the inner product, and

(ii) $R_{g}^{*} \xi=\rho\left(g^{-1}\right) \xi$ for $g \in G$.

We will be interested only in tensorial forms of the type $(g, a d)$, so henceforth we shall use the words tensorial form to mean tensorial form of the type $(8, a d)$.

Given a connection form $\omega$ on $P$, we define the exterior covariant differentiation $D$ on the tensorial forms by $D \alpha=d \alpha+[\omega, \alpha]$.

The space of tensorial forms is stable under the action $D$. It is also easy to see that the curvature form $\Omega$ is a tensorial 2 -form. If $\omega_{0}, \omega_{1}$ are two connec- 
tion forms on $P$, then $\omega_{1}-\omega_{0}$ is a tensorial form.

We prepare the following well-known lemma which will be used in the sequel.

LEMMA (1.2). (i) $D \Omega=0$ (Bianchi identity),

(ii) $D^{2} \alpha=[\Omega, \alpha]$ if $\alpha$ is a tensorial form.

Proof.

$$
\begin{aligned}
D \Omega & =d \Omega+[\omega, \Omega]=d(d \omega+1 / 2[\omega, \omega])+[\omega, d \omega+1 / 2[\omega, \omega]] \\
& =[d \omega, \omega]+[\omega, d \omega] \text { by Jacobi identity } \\
& =0 \text { by }(1.1) . \\
D^{2} \alpha & =d(D \alpha)+[\omega, D \alpha]=d(d \alpha+[\omega, \alpha])+[\omega, d \alpha+[\omega, \alpha]] \\
& =[d \omega, \alpha]+[\omega,[\omega, \alpha]] \\
& =[d \omega, \alpha]+1 / 2[[\omega, \omega], \alpha] \text { by }(1.3) \\
& =[\Omega, \alpha] .
\end{aligned}
$$

Denote by $I^{k}(G)$ the set of all symmetric multilinear mappings $\varphi: g \times \cdots$ $\times 8 \rightarrow R$ such that $\varphi\left(\operatorname{ad}(g) X_{1}, \ldots, \operatorname{ad}(g) X_{k}\right)=\varphi\left(X_{1}, \ldots, X_{k}\right)$ for $g \in G, X_{i} \in \mathbf{g}$.

If $\xi_{1}, \ldots, \xi_{k}$ are $g$-valued forms on $P$ of degree $d_{1}, \ldots, d_{k}$ respectively, then we can define a $\left(d_{1}+d_{2}+\cdots+d_{k}\right)$-form $\varphi\left(\xi_{1}, \ldots, \xi_{k}\right)$ on $P$ as follows:

If $\left\{e_{i}\right\}_{i=1}^{n}$ is a basis of $g, \xi_{i}=\Sigma_{j} \xi_{i}^{j} \otimes e_{j}$ for $i=1,2, \ldots, k$

$$
\varphi\left(\xi_{1}, \ldots, \xi_{k}\right)=\sum \varphi\left(e_{j_{1}}, \ldots, e_{j_{k}}\right) \xi_{1}^{j_{1}} \wedge \cdots \wedge \xi_{k}^{j_{k}} \text {. }
$$

The definition is independent of the choice of $e_{1}, \ldots, e_{n}$. If $\left\{\xi_{i}\right\}_{i=1}^{k}$ are tensorial forms, then the form $\varphi\left(\xi_{1}, \ldots, \xi_{k}\right)$ can be projected to the base manifold $M$. The following proposition is also well known.

Proposition (1.3). (i) Let $\xi_{i}$ be a tensorial $d_{i}$-form on $P$. Then for $\varphi \in$ $I^{k}(G)$, we have

$$
d \varphi\left(\xi_{1}, \ldots, \xi_{k}\right)=\sum(-1)^{d_{1}+\cdots+d_{i-1}} \varphi\left(\xi_{1}, \ldots, D \xi_{i}, \ldots, \xi_{k}\right)
$$

for any connection $D$.

(ii) For 8 -valued 1-form $\theta$ on $P$, we have

$$
\sum(-1)^{d_{1}+\cdots+d_{i}} \varphi\left(\xi_{1}, \ldots,\left[\xi_{i}, \theta\right], \ldots, \xi_{k}\right)=0 .
$$

For convenience, we will use the following notation used by Chern. For 
$\varphi \in I^{k}(G), \varphi\left(X_{1}, \ldots, X_{l} ; Y\right)$ abbreviates the function that $Y$ appears in the last $(k-l)$-slots. In particular, $\varphi(X)=\varphi(X, \ldots, X), \varphi(X ; Y)=\varphi(X, Y, \ldots, Y)$.

Let $P$ be a principal $G$-bundle over $M$ with connection form $\omega$ on $P$. Put $\omega_{t}=t \omega, t \in[0,1], \Omega_{t}=d \omega_{t}+1 / 2\left[\omega_{t}, \omega_{t}\right]$.

For $\varphi \in I^{k}(G)$, we define

$$
T \varphi(\omega)=k \int_{0}^{1} \varphi\left(\omega ; \Omega_{t}\right) d t
$$

Then we have $d T \varphi(\omega)=\varphi(\Omega)$. The dependence of this form on the connections is given by the following.

Proposition (1.4) (CHERnSimons [3]). If $\omega_{s}$ is a 1-parameter family of connections on $P$, then

$$
(\partial / \partial s) T \varphi\left(\omega_{s}\right)=k \varphi\left((\partial / \partial s) \omega_{s} ; \Omega_{s}\right)+\text { exact form. }
$$

A simple proof of this formula is given in $\S 4$.

2. The main formula. If $\omega_{0}$ and $\omega_{1}$ are two connection forms on $P$, put $\alpha=\omega_{1}-\omega_{0}, \omega_{t}=\omega_{0}+t \alpha, t \in[0,1], \Omega_{t}=d \omega_{t}+1 / 2\left[\omega_{t}, \omega_{t}\right]$.

Then $\alpha, \Omega_{t}$ are tensorial forms and we have the following generalized Gauss-Codazzi equation.

$$
\Omega_{t}=\Omega_{0}+t D \alpha+\left(t^{2} / 2\right)[\alpha, \alpha] \text { for } t \in[0,1],
$$

where $D$ is the exterior covariant derivative with respect to the connection form $\omega_{0}$.

PROOF OF (2.1).

$$
\begin{aligned}
\Omega_{t} & =d \omega_{t}+1 / 2\left[\omega_{t}, \omega_{t}\right]=d \omega_{0}+t d \alpha+1 / 2\left[\omega_{0}+t \alpha, \omega_{0}+t \alpha\right] \\
& =\Omega_{0}+t\left(d \alpha+\left[\omega_{0}, \alpha\right]\right)+\left(t^{2} / 2\right)[\alpha, \alpha]=\Omega_{0}+t D \alpha+\left(t^{2} / 2\right)[\alpha, \alpha] .
\end{aligned}
$$

Similarly, we have

$$
\Omega_{t}=t \Omega_{1}+(1-t) \Omega_{0}+\frac{t(t-1)}{2}[\alpha, \alpha] \text { for } t \in[0,1] \text {. }
$$

Comparing (2.1) and (2.2), we have

$$
D \alpha=\Omega_{1}-\Omega_{0}-1 / 2[\alpha, \alpha] .
$$

Instead of the Bianchi identity, we have

$$
D \Omega_{t}=t\left[\Omega_{t}, \alpha\right] \text {. }
$$

Proof of (2.4). By the Bianchi identity, we have $D_{t} \Omega_{t}=0 . D_{t}$, by definition, is the exterior covariant derivative with respect to $\omega_{t}$. Hence

$$
0=D_{t} \Omega_{t}=d \Omega_{t}+\left[\omega_{t}, \Omega_{t}\right]=d \Omega_{t}+\left[\omega_{0}+t \alpha, \Omega_{t}\right]=D \Omega_{t}+t\left[\alpha, \Omega_{t}\right] .
$$


Thus, by (1.1), we obtain (2.4).

The following proposition is well known.

Proposition (2.1) (Chern-Weil). If $\varphi \in I^{k}(G)$, then $(\partial / \partial t) \varphi\left(\Omega_{t}\right)=$ $d\left(k \varphi\left(\alpha ; \Omega_{t}\right)\right)$.

The proof of this proposition can be found in [5].

Motivated by Chern-Simons' work, we propose to study the dependence of the form $\varphi\left(\alpha ; \Omega_{t}\right)$ on the connections.

MAIN Formula (2.5). Let $\omega, \omega_{s}, s \in[0,1]$, be connection forms on $P$. Put

$$
\alpha_{s}=\omega_{s}-\omega, \quad \omega_{s}^{t}=\omega+t \alpha_{s}, \quad \Omega_{s}^{t}=d \omega_{s}^{t}+1 / 2\left[\omega_{s}^{t}, \omega_{s}^{t}\right] .
$$

Then for $\varphi \in I^{k}(G)$, we have

$$
\begin{aligned}
\frac{\partial}{\partial s} \varphi\left(\alpha_{s} ; \Omega_{s}^{t}\right)= & -(k-1) t d \varphi\left(\alpha_{s}, \frac{\partial}{\partial s} \omega_{s} ; \Omega_{s}^{t}\right) \\
& +\varphi\left(\frac{\partial}{\partial s} \omega_{s}, \Omega+k t D \alpha_{s}+\frac{t^{2}}{2}(2 k-1)\left[\alpha_{s}, \alpha_{s}\right] ; \Omega_{s}^{t}\right),
\end{aligned}
$$

where $D$ is the exterior covariant derivative with respect to the connection $\omega$.

Proof.

$$
\begin{aligned}
\frac{\partial}{\partial s} \varphi\left(\alpha_{s} ; \Omega_{s}^{t}\right)+(k-1) t d \varphi\left(\alpha_{s}, \frac{\partial}{\partial s} \omega_{s} ; \Omega_{s}^{t}\right) \\
=\varphi\left(\frac{\partial}{\partial s} \alpha_{s} ; \Omega_{s}^{t}\right)+(k-1) \varphi\left(\alpha_{s}, \frac{\partial}{\partial s} \Omega_{s}^{t} ; \Omega_{s}^{t}\right) \\
\quad+(k-1) t \varphi\left(D \alpha_{s}, \frac{\partial}{\partial s} \omega_{s} ; \Omega_{s}^{t}\right)-(k-1) t \varphi\left(\alpha_{s}, \frac{\partial}{\partial s} D \alpha_{s} ; \Omega_{s}^{t}\right) \\
\quad+(k-1)(k-2) t \varphi\left(\alpha_{s}, \frac{\partial}{\partial s} \omega_{s}, D \Omega_{s}^{t} ; \Omega_{s}^{t}\right) .
\end{aligned}
$$

By the generalized Gauss-Codazzi equation (2.1), we have $\Omega_{s}^{t}=\Omega+t D \alpha_{s}+$ $\left(t^{2} / 2\right)\left[\alpha_{s}, \alpha_{s}\right]$. Hence we have

$$
\frac{\partial}{\partial s} \Omega_{s}^{t}=t \frac{\partial}{\partial s} D \alpha_{s}+t^{2}\left[\frac{\partial}{\partial s} \alpha_{s}, \alpha_{s}\right] .
$$

Thus the second and fourth terms of the right-hand side yield to

$$
\begin{aligned}
(k-1) \varphi\left(\alpha_{s} ; \frac{\partial}{\partial s} \Omega_{s}^{t}\right) & -t(k-1) \varphi\left(\alpha_{s}, \frac{\partial}{\partial s} D \alpha_{s} ; \Omega_{s}^{t}\right) \\
= & (k-1) \varphi\left(\alpha_{s}, t^{2}\left[\frac{\partial}{\partial s} \alpha_{s}, \alpha_{s}\right] ; \Omega_{s}^{t}\right) .
\end{aligned}
$$


By the invariance of $\varphi$, Proposition (1.3), together with (2.4), we can simplify the right-hand side to

$$
\begin{aligned}
R H S= & \varphi\left(\frac{\partial}{\partial s} \alpha_{s} ; \Omega_{s}^{t}\right)+(k-1) t^{2} \varphi\left(\alpha_{s},\left[\frac{\partial}{\partial s} \alpha_{s}, \alpha_{s}\right] ; \Omega_{s}^{t}\right) \\
& +(k-1) t \varphi\left(D \alpha_{s}, \frac{\partial}{\partial s} \alpha_{s} ; \Omega_{s}^{t}\right)+(k-1)(k-2) t \varphi\left(\alpha_{s}, \frac{\partial}{\partial s} \alpha_{s}, D \Omega_{s}^{t} ; \Omega_{s}^{t}\right) \\
= & \varphi\left(\frac{\partial}{\partial s} \alpha_{s} ; \Omega_{s}^{t}\right)+(k-1) t^{2} \varphi\left(\left[\alpha_{s}, \alpha_{s}\right], \frac{\partial}{\partial s} \alpha_{s} ; \Omega_{s}^{t}\right) \\
& +(k-1) t \varphi\left(\frac{\partial}{\partial s} \alpha_{s}, D \alpha_{s} ; \Omega_{s}^{t}\right) \\
= & \varphi\left(\frac{\partial}{\partial s} \alpha_{s}, \Omega_{s}^{t}+(k-1) t^{2}\left[\alpha_{s}, \alpha_{s}\right]+(k-1) t D \alpha_{s} ; \Omega_{s}^{t}\right) \\
= & \varphi\left(\frac{\partial}{\partial s} \alpha_{s}, \Omega+t k D \alpha_{s}+\frac{t^{2}}{2}\left[\alpha_{s}, \alpha_{s}\right] ; \Omega_{s}^{t}\right) \text { by (2.1). Q.E.D. }
\end{aligned}
$$

Integrating the main formula, we have

COROllary (2.2) (HeITSCH) [4]. The assumptions are the same as in the main formula. We have

$$
\frac{\partial}{\partial s} \int_{0}^{1} \varphi\left(\alpha_{s} ; \Omega_{s}^{t}\right) d t=\varphi\left(\frac{\partial}{\partial s} \alpha_{s} ; \Omega_{s}\right)+\text { exact form }
$$

In $\S 4$, we will see that this formula can be derived quite easily from a more general setting. As an application, we have the generalized Chern-Weil formula.

COROLlaRY (2.3). Let $\omega_{s}$ be a smooth 1-parameter family of connections on $P$. Then for $\varphi \in I^{k}(G)$ we have

$$
(\partial / \partial s) \varphi\left(\Omega_{s}\right)=d\left(k \varphi\left((\partial / \partial s) \omega_{s} ; \Omega_{s}\right)\right)
$$

Another application of the main formula is concerned with flat connections.

Proposition (2.4). Let $\omega$ be a flat connection in the main formula (2.5). Then for $\varphi \in I^{k}(G)$, we have

$$
\frac{\partial}{\partial s} \varphi\left(\alpha_{s} ; D \alpha_{s}\right)=-(k-1) d \varphi\left(\alpha_{s}, \frac{\partial}{\partial s} \alpha_{s} ; D \alpha_{s}\right)+k \varphi\left(\frac{\partial}{\partial s} \alpha_{s} ; D \alpha_{s}\right)
$$

Proof. Since $\omega$ is flat, by (2.1) we have $\Omega_{s}^{t}=t D \alpha_{s}+\left(t^{2} / 2\right)\left[\alpha_{s}, \alpha_{s}\right]$. Putting $\Omega_{s}^{t}$ in the main formula, we have 


$$
\begin{gathered}
\frac{\partial}{\partial s} \varphi\left(\alpha_{s} ; t D \alpha_{s}+\frac{t^{2}}{2}\left[\alpha_{s}, \alpha_{s}\right]\right)=-(k-1) t d \varphi\left(\alpha_{s}, \frac{\partial}{\partial s} \alpha_{s} ; t D \alpha_{s}+\frac{t^{2}}{2}\left[\alpha_{s}, \alpha_{s}\right]\right) \\
+\varphi\left(\frac{\partial}{\partial s} \alpha_{s}, k t D \alpha_{s}+\frac{t^{2}}{2}(2 k-1)\left[\alpha_{s}, \alpha_{s}\right] ; t D \alpha_{s}+\frac{t^{2}}{2}\left[\alpha_{s}, \alpha_{s}\right]\right) .
\end{gathered}
$$

Comparing the coefficients of $t^{k-1}$, we obtain

$$
\frac{\partial}{\partial s} \varphi\left(\alpha_{s} ; D \alpha_{s}\right)=-(k-1) d \varphi\left(\alpha_{s}, \frac{\partial}{\partial s} \alpha_{s} ; D \alpha_{s}\right)+k \varphi\left(\frac{\partial}{\partial s} \alpha_{s} ; D \alpha_{s}\right) \text {. Q.E.D. }
$$

If $\omega_{0}$ is flat, then from Proposition (2.1), we have $\varphi\left(\Omega_{1}\right)=d\left(k \int_{0}^{1} \varphi\left(\alpha ; \Omega_{t}\right) d t\right)$. Hence if $\varphi\left(\Omega_{1}\right)=0$, then the form $\Delta_{\varphi}\left(\omega_{0}, \omega_{1}\right)=k \int_{0}^{1} \varphi\left(\alpha ; \Omega_{t}\right) d t$ is a closed form on $M$, and we have the following:

THEOREM 1. If $\varphi \in I^{k}(G)$ and $\omega_{1}$ is any connection form on $P$ such that $\varphi\left(\Omega_{1}\right)=0$, then for any flat connection $\omega_{0}$ and $k>1$, the cohomology class $\left\{\Delta_{\varphi}\left(\omega_{0}, \omega_{1}\right)\right\} \in H^{2 k-1}(M ; R)$ is a deformation invariant, that is, an invariant through the deformations of flat connections.

Let $P$ be a flat $\mathrm{GL}(m)$-bundle over $M$ with flat connection $\omega_{0}$. If $Q$ is an $O(m)$-reduction, then for any connection $\omega_{1}$ on $Q, \varphi\left(\Omega_{1}\right)=0$, where $\operatorname{deg} \varphi=$ $2 k-1(k=1,2,3, \ldots)$. In this case, the class $\left\{\Delta_{\varphi}\left(\omega_{0}, \omega_{1}\right)\right\} \in H^{4 k-3}(M)$ is the same as the secondary characteristic invariants $\Delta_{\varphi}\left(x_{k}\right) \in H^{4 k-3}(M)(k=1,2$, ... ) constructed by Kamber and Tondeur [7]. See [8, p. 69] for the existence and $[8, \mathrm{p} .86]$ for the deformation invariance for $k>1$ of these invariants.

3. Characteristic classes for flat connections. Let $\omega_{0}, \omega_{1}$ be two flat connections of the $G$-bundle $P$ over $M$. Put $\alpha=\omega_{1}-\omega_{0}$; then for $\varphi \in I^{k}(G)$, $\varphi(\alpha ; D \alpha)$ is a $(2 k-1)$-form on $P . \varphi(\alpha ; D \alpha)$ can be projected to the base manifold $M$, that is, there exists a unique $(2 k-1)$-form $\xi$ on $M$ such that its pullback by the projection is $\varphi(\alpha ; D \alpha)$.

Proposition (3.1). If $k>1$, $\xi$ is a closed form.

Proof. It suffices to prove that $\varphi(\alpha ; D \alpha)$ is a closed form. From Lemma (1.2) and the assumption that $\omega_{0}$ is flat, we have $D^{2} \alpha=0$. By (2.3), we obtain $D \alpha=-1 / 2[\alpha, \alpha]$. Thus $d \varphi(\alpha ; D \alpha)=\varphi(D \alpha)=(-1 / 2)^{k} \varphi([\alpha, \alpha])$. By the invariance of $\varphi$, we have

$$
\varphi([\alpha, \alpha])+(k-1) \varphi(\alpha,[[\alpha, \alpha], \alpha] ;[\alpha, \alpha])=0 .
$$

Therefore we conclude that $d \varphi(\alpha ; D \alpha)=0$ by (1.4).

We will exhibit a formula to see how this form depends on the connections.

Proposition (3.2). Suppose $\omega, \omega_{s}, s \in[0,1]$, are flat connections on $P$. Then for $\varphi \in I^{k}(G), k>1$, we have 


$$
\frac{\partial}{\partial s} \varphi\left(\alpha_{s} ; D \alpha_{s}\right)=-(2 k-1) d \varphi\left(\alpha_{s}, \frac{\partial}{\partial s} \alpha_{s} ; D \alpha_{s}\right)
$$

Proof.

$$
\begin{aligned}
\frac{\partial}{\partial s} \varphi\left(\alpha_{s} ; D \alpha_{s}\right) & =\varphi\left(\frac{\partial}{\partial s} \alpha_{s} ; D \alpha_{s}\right)+(k-1) \varphi\left(\alpha_{s}, \frac{\partial}{\partial s} D \alpha_{s} ; D \alpha_{s}\right) \\
& =\varphi\left(\frac{\partial}{\partial s} \alpha_{s} ; D \alpha_{s}\right)+(k-1) \varphi\left(\alpha_{s},-\left[\frac{\partial}{\partial s} \alpha_{s}, \alpha_{s}\right] ; D \alpha_{s}\right) \\
& =\varphi\left(\frac{\partial}{\partial s} \alpha_{s} ; D \alpha_{s}\right)-(k-1) \varphi\left(\left[\alpha_{s}, \alpha_{s}\right], \frac{\partial}{\partial s} \alpha_{s} ; D \alpha_{s}\right) \\
& =\varphi\left(\frac{\partial}{\partial s} \alpha_{s} ; D \alpha_{s}\right)+2(k-1) \varphi\left(\frac{\partial}{\partial s} \alpha_{s} ; D \alpha_{s}\right) \\
& =(2 k-1) \varphi\left(\frac{\partial}{\partial s} \alpha_{s} ; D \alpha_{s}\right) .
\end{aligned}
$$

Comparing this equality with Proposition (2.4), we have

$$
\frac{\partial}{\partial s} \varphi\left(\alpha_{s} ; D \alpha_{s}\right)=-(2 k-1) d \varphi\left(\alpha_{s}, \frac{\partial}{\partial s} \alpha_{s} ; D \alpha_{s}\right)
$$

As a consequence, we have the following

THEOREM 2. For $\varphi \in I^{k}(G),\{\varphi(\alpha ; D \alpha)\} \in H^{2 k-1}(M ; R)$ is a deformation invariant for $k>1$.

Proof. From Proposition (3.2), we have

$$
\frac{\partial}{\partial s} \varphi\left(\alpha_{s} ; D \alpha_{s}\right)=-(2 k-1) d \varphi\left(\alpha_{s}, \frac{\partial}{\partial s} \alpha_{s} ; D \alpha_{s}\right) \text {. }
$$

Integrating both sides, we have

$$
\varphi\left(\alpha_{1} ; D \alpha_{1}\right)-\varphi\left(\alpha_{0} ; D \alpha_{0}\right)=d\left(-(2 k-1) \int_{0}^{1} \varphi\left(\alpha_{s}, \frac{\partial}{\partial s} \alpha_{s} ; D \alpha_{s}\right) d s\right) \text {. Q.E.D. }
$$

In the assumptions of Proposition (3.2), if we put $\omega=\omega_{0}$, then $\alpha_{0}=0$. Hence $\varphi\left(\alpha_{1} ; D \alpha_{1}\right)$ is an exact form. Therefore this class gives an obstruction to the deformations of flat connections. We state this fact in the following

Proposition (3.3). If $\omega_{s}, s \in[0,1]$, is a smooth 1-parameter family of flat connections, put $\alpha=\omega_{1}-\omega_{0}$. Then for $\varphi \in I^{k}(G), k>1$, we have $\{\varphi(\alpha ; D \alpha)\}=0$ in $H^{2 k-1}(M ; R)$.

The following proposition given by Kamber and Tondeur in [7] will show that Proposition (3.3) is false for $k=1$.

Let $M$ be a manifold with flat connection $\omega_{0}$ and an arbitrary Riemannian flat connection $\omega_{1}$. Such a manifold exists, for example $M=T^{2}$. Let $P$ be the frame bundle of $M$. 
Proposition (3.4) (Kamber-Tondeur). Suppose $\operatorname{dim} M=n \geqslant 2$. If $h: \pi_{1}(M) \rightarrow \Gamma$ is the holonomy representation of the connection $\omega_{0}$, then we have

$$
\int_{\gamma} \operatorname{tr} \omega_{0}=-\frac{1}{2 \pi} \log |\operatorname{det} h(\gamma)| \text { for } \gamma \in \pi_{1}(M) .
$$

We will give an example to show these classes are nontrivial.

Let $M$ be the group manifold $S O(3)$. Denote by $P$ the frame bundle over $M$ with structure group $\mathrm{GL}(3 ; R)$. The Lie algebra of $\mathrm{GL}(3 ; R)$ is $\operatorname{gl}(3 ; R)$. Let $e_{i j}$ denote the matrix in $g l(3 ; R)$ with the entry in the $i$ th row and $j$ th column equal to 1 and all other entries 0 .

Put $X_{1}=e_{12}-e_{21}, X_{2}=e_{13}-e_{31}, X_{3}=e_{23}-e_{32} .\left\{X_{i}\right\}_{i=1}^{3}$ is a basis of $80(3)$, the Lie algebra of $S O(3)$. We have

$$
\left[X_{1}, X_{2}\right]=-X_{3},\left[X_{3}, X_{1}\right]=-X_{2},\left[X_{2}, X_{3}\right]=-X_{1} \text {. }
$$

If we denote by $\left\{\theta^{i}\right\}_{i=1}^{3}$ the left invariant forms on $S O(3)$ dual to the basis $\left\{X_{i}\right\}_{i=1}^{3}$, then the Maurer-Cartan equations are given by

$$
d \theta^{1}=\theta^{2} \wedge \theta^{3}, d \theta^{2}=\theta^{3} \wedge \theta^{1}, d \theta^{3}=\theta^{1} \wedge \theta^{2} .
$$

The $(+)$ connection on $S O(3)$ is defined by $\nabla_{X} Y=[X, Y]$ for all left invariant vector fields $X, Y$. Denote by $\omega_{0}$ the connection form with respect to $\left\{X_{i}\right\}_{i=1}^{3}$, that is, $\nabla X_{i}=\left(\omega_{0}\right)_{i}^{j} \otimes X_{j}$. We have

$$
\omega_{0}=\left(\begin{array}{ccc}
0 & \theta^{3} & -\theta^{2} \\
-\theta^{3} & 0 & \theta^{1} \\
\theta^{2} & -\theta^{1} & 0
\end{array}\right) .
$$

We know that $\omega_{0}$ is flat. The $(-)$ connection is defined by $\nabla X=0$ for all left invariant vector fields. If we denote by $\omega_{1}$ its connection form, then $\omega_{1}=$ 0. Again, $\omega_{1}$ is also flat.

Put $\alpha=\omega_{1}-\omega_{0}$ and let $D \alpha$ be the exterior covariant derivative of $\alpha$ with respect to the connection $\omega_{0}$. Then

$$
D \alpha=\left(\begin{array}{cccc}
0 & \theta^{1} \wedge \theta^{2} & -\theta^{3} \wedge \theta^{1} \\
-\theta^{1} \wedge \theta^{2} & 0 & \theta^{2} \wedge \theta^{3} \\
\theta^{3} \wedge \theta^{1} & -\theta^{2} \wedge \theta^{3} & 0
\end{array}\right) .
$$

If we consider the invariant polynomial $\varphi \in I^{2}(\mathrm{GL}(3 ; R))$ defined by $\varphi(X)$ $=\operatorname{trace}\left(X^{2}\right)$, then $\varphi(\alpha ; D \alpha)=6 \theta^{1} \wedge \theta^{2} \wedge \theta^{3}$. Hence $\{\varphi(\alpha ; D \alpha)\} \neq 0$ in $H^{3}(S O(3) ; R)$, and we have

Proposition (3.5). The $(+)$ and $(-)$ connections in $S O(3)$ cannot be deformed to each other.

As will be seen in $\S 4, T \varphi\left(\omega_{0}\right)$ and $T \varphi\left(\omega_{1}\right)$ define different cohomology 
classes. By virtue of Theorem 3, we again have the nondeformability of (+) and $(-)$ connections.

4. Generalized Chern-Weil formula. We will generalize Bott's work in [1] and give a more systematic treatment of the transgression forms.

Let $P$ be a principal $G$-bundle over $M$ with projection $\pi . P \times R^{n}$ is then a $G$-bundle over $M \times R^{n}$ with projection $\pi \times$ id.

Given $\varphi \in I^{k}(G)$ and a connection form $\omega$ on $P$, we define two forms $\mu(\omega) \varphi$ and $\lambda(\omega) \varphi$ on $P$ of degree $2 k-1$ and $2 k$ respectively by

$$
\mu(\omega) \varphi=T \varphi(\omega), \quad \lambda(\omega) \varphi=\varphi(\Omega) .
$$

Then we have $d(\mu(\omega) \varphi)=\lambda(\omega) \varphi$.

If $\omega_{0}, \omega_{1}, \ldots, \omega_{n}$ are connection forms on $P$, define a connection $\omega_{0,1,2, \ldots, n}$ on the principal $G$-bundle $P \times R^{n} \rightarrow M \times R^{n}$ as follows,

$$
\omega_{0,1,2, \ldots, n}=\left\{\begin{array}{l}
\left(1-\Sigma_{i=1}^{n} a_{i}\right) \omega_{0}+a_{1} \omega_{1}+\cdots+a_{n} \omega_{n} \text { on } P \times\left\{a_{i}\right\}_{i=1}^{n}, \\
0 \text { on }\{u\} \times R^{n} \text { for } u \in P .
\end{array}\right.
$$

Let $\Delta^{n}=\left\{\left(a_{1}, \ldots, a_{n}\right) \in R^{n}, \Sigma_{i=1}^{n} a_{i} \leqslant 1, a_{i} \geqslant 0\right\}$. Then $P \times \Delta^{n} \underset{\pi_{1}}{\longrightarrow}$ $P$ is a fibre bundle with fibre $\Delta^{n}$ a compact space. Thus we have the notion of integration along the fibre $\pi_{1}$. More precisely, $\pi_{1}$ is a canonical homomorphism $\pi_{1}: \Lambda^{r}\left(P \times \Delta^{n}\right) \rightarrow \Lambda^{r-n}(P)$ which is zero for $r<n$ and satisfying

$$
d \circ \pi_{1 *}+(-1)^{n+1} \pi_{1 *} \circ d=(-1)^{n+1} \pi_{1 *}^{\partial} \circ i^{*}
$$

where $\pi_{1}^{\partial}$ is the projection of the fibre bundle $\pi_{1}^{\partial}: P \times \partial \Delta^{n} \rightarrow P$.

We define two differential forms

$$
\lambda\left(\omega_{0}, \omega_{1}, \ldots, \omega_{n}\right) \varphi \text { and } \mu\left(\omega_{0}, \omega_{1}, \ldots, \omega_{n}\right) \varphi
$$

on $P$ by

$$
\begin{aligned}
& \lambda\left(\omega_{0}, \omega_{1}, \ldots, \omega_{n}\right) \varphi=(-1)^{[n / 2]} \pi_{1}\left(\left.\lambda\left(\omega_{0,1,2, \ldots, n}\right) \varphi\right|_{P \times \Delta^{n}}\right), \\
& \mu\left(\omega_{0}, \omega_{1}, \ldots, \omega_{n}\right) \varphi=(-1)^{[n / 2]} \pi_{1}\left(\left.\mu\left(\omega_{0,1,2, \ldots, n}\right) \varphi\right|_{P \times \Delta^{n}}\right)
\end{aligned}
$$

we have

$$
\begin{aligned}
& \operatorname{deg} \lambda\left(\omega_{0}, \omega_{1}, \ldots, \omega_{n}\right) \varphi=2 k-n, \\
& \operatorname{deg} \mu\left(\omega_{0}, \omega_{1}, \ldots, \omega_{n}\right) \varphi=2 k-1-n .
\end{aligned}
$$

Notice that $\lambda\left(\omega_{0}, \omega_{1}, \ldots, \omega_{n}\right) \varphi$ is a form on $M$, while $\mu\left(\omega_{0}, \omega_{1}, \ldots, \omega_{n}\right) \varphi$ is defined, in general, only on $P$.

The relations among these forms are given by 


$$
\begin{aligned}
d \mu\left(\omega_{0}, \omega_{1}, \ldots, \omega_{n}\right) \varphi= & (-1)^{n} \lambda\left(\omega_{0}, \omega_{1}, \ldots, \omega_{i}, \ldots, \omega_{n}\right) \varphi \\
& +\sum_{i=0}^{n}(-1)^{i} \mu\left(\omega_{0}, \omega_{1}, \ldots, \hat{\omega}_{i}, \ldots, \omega_{n}\right) \varphi
\end{aligned}
$$

where ${ }^{\wedge}$ means the deleted term.

Proof.

$$
\begin{aligned}
d \mu\left(\omega_{0}, \omega_{1}, \ldots, \omega_{n}\right) \varphi=(-1)^{[n / 2]} d \pi_{1},\left(\left.\mu\left(\omega_{0,1,2, \ldots, n}\right) \varphi\right|_{P \times \Delta^{n}}\right) \\
=(-1)^{[n / 2]}(-1)^{n} \pi_{1}\left(\left.d \mu\left(\omega_{0,1,2, \ldots, n}\right) \varphi\right|_{P \times \Delta^{n}}\right) \\
+(-1)^{[n / 2]}(-1)^{n+1} \pi_{1}^{\partial}\left(\left.\mu\left(\omega_{0,1,2, \ldots, n}\right) \varphi\right|_{P \times \partial \Delta^{n}}\right) \text { by (4.1). }
\end{aligned}
$$

The first term in the right-hand side is $(-1)^{n} \lambda\left(\omega_{0}, \omega_{1}, \ldots, \omega_{n}\right) \varphi_{1}$ by the fact that $d \mu(\omega) \varphi=\lambda(\omega) \varphi$. By the definition of connection $\omega_{0,1,2, \ldots, n}$, we see that

$$
\begin{aligned}
& \pi_{1_{*}}^{\partial}\left(\left.\mu\left(\omega_{0,1,2, \ldots, n}\right) \varphi\right|_{P \times \partial \Delta^{n}}\right) \\
& \quad=\pi_{1 *}\left(\left.\sum_{i=1}^{n}(-1)^{i} \mu\left(\omega_{0,1,2, \ldots, \hat{i}, \ldots, n}\right) \varphi\right|_{P \times \Delta^{n-1}}\right) \\
& \quad=(-1)^{[(n-1) / 2]} \sum_{i=0}^{n}(-1)^{i} \mu\left(\omega_{0}, \omega_{1}, \ldots, \hat{\omega}_{i}, \ldots, \omega_{n}\right) \varphi .
\end{aligned}
$$

Going back to the original identity, we have

$$
\begin{aligned}
& d \mu\left(\omega_{0}, \omega_{1}, \ldots, \omega_{n}\right) \varphi \\
& =(-1)^{n} \lambda\left(\omega_{0}, \omega_{1}, \ldots, \omega_{n}\right) \varphi \\
& \quad+(-1)^{[n / 2]+(n+1)+[(n-1) / 2]} \sum_{i=0}^{n}(-1)^{i} \mu\left(\omega_{0}, \omega_{1}, \ldots, \omega_{i}, \ldots, \omega_{n}\right) \varphi .
\end{aligned}
$$

By the fact that $[n / 2]+(n+1)+[(n-1) / 2]$ is always an even integer, we have (4.2).

From this formula, we can recover the formula given by Bott in [1].

$$
d \lambda\left(\omega_{0}, \omega_{1}, \ldots, \omega_{n}\right) \varphi=\sum_{i=0}^{n}(-1)^{i} \lambda\left(\omega_{0}, \omega_{1}, \ldots, \hat{\omega}_{i}, \ldots, \omega_{n}\right) \varphi .
$$

Proof of (4.3). Applying the exterior differential operator $d$ to both sides of (4.2), we have

$$
0=(-1)^{n} d \lambda\left(\omega_{0}, \omega_{1}, \ldots, \omega_{n}\right) \varphi+\sum_{i=0}^{n}(-1)^{i} d \mu\left(\omega_{0}, \omega_{1}, \ldots, \hat{\omega}_{i}, \ldots, \omega_{n}\right) \varphi
$$

If we apply the formula (4.2) again, we have 


$$
\begin{aligned}
& d \lambda\left(\omega_{0}, \omega_{1}, \ldots, \omega_{n}\right) \varphi=(-1)^{n+1} \sum_{i=0}^{n}(-1)^{i} d \mu\left(\omega_{0}, \ldots, \hat{\omega}_{i}, \ldots, \omega_{n}\right) \varphi \\
&=(-1)^{n+1} \sum_{i=0}^{n}(-1)^{i}(-1)^{n-1} \lambda\left(\omega_{0}, \omega_{1}, \ldots, \hat{\omega}_{i}, \ldots, \omega_{n}\right) \varphi \\
&+(-1)^{n+1} \sum_{j<i}(-1)^{i+j} \mu\left(\omega_{0}, \omega_{1}, \ldots, \hat{\omega}_{j}, \ldots, \hat{\omega}_{i}, \ldots, \omega_{n}\right) \varphi \\
&+(-1)^{n+1} \sum_{j>i}(-1)^{i+j+1} \mu\left(\omega_{0}, \omega_{1}, \ldots, \hat{\omega}_{i}, \ldots, \hat{\omega}_{j}, \ldots, \omega_{n}\right) \varphi
\end{aligned}
$$

The second and third terms in the right-hand side cancel each other, so we obtain (4.3).

Before proceeding any further, we will explain these forms in the case $n=1$.

$$
\lambda\left(\omega_{0}, \omega_{1}\right) \varphi=k \int_{0}^{1} \varphi\left(\omega_{1}-\omega_{0} ; \Omega_{t}\right) d t,
$$

where $\Omega_{t}$ is the curvature form of the connection $\omega_{t}=t \omega_{1}+(1-t) \omega_{0}$ for $t \in[0,1]$. In fact, if we denote the curvature form of the connection $\omega_{0,1}$ by $\Omega_{0,1}$, we have

$$
\begin{aligned}
\lambda\left(\omega_{0}, \omega_{1}\right) \varphi & =\pi_{1_{*}}\left(\left.\lambda\left(\omega_{0,1}\right) \varphi\right|_{P \times \Delta^{1}}\right)=\pi_{1 *}\left(\left.\varphi\left(\Omega_{0,1}\right)\right|_{P \times \Delta^{1}}\right) \\
& =\pi_{1} \varphi\left(d t \wedge\left(\omega_{1}-\omega_{0}\right)+\Omega_{t}\right) \text { on } P \times\{t\} \\
& =k \int_{0}^{1} \varphi\left(\omega_{1}-\omega_{0} ; \Omega_{t}\right) d t .
\end{aligned}
$$

$$
\mu\left(\omega_{0}, \omega_{1}\right) \varphi=k(k-1) \int_{0}^{1} \int_{0}^{1} \varphi\left(\omega_{t}, s \alpha ; s \Omega_{t}+\frac{s^{2}-s}{2}[\alpha, \alpha]\right) d s d t
$$

where $\alpha=\omega_{1}-\omega_{0}, \omega_{t}=t \omega_{1}+(1-t) \omega_{0}$ for $t \in[0,1], \Omega_{t}=d \omega_{t}+$ $1 / 2\left[\omega_{t}, \omega_{t}\right]$.

ProOF OF (4.5).

$$
\begin{aligned}
\mu\left(\omega_{0}, \omega_{1}\right) \varphi & \left.=\pi_{1},\left.\mu\left(\omega_{0,1}\right) \varphi\right|_{P \times \Delta_{1}^{1}}\right) \\
& =\pi_{1 *} k \int_{0}^{1} \varphi\left(\omega_{0,1} ; s d \omega_{0,1}+\frac{s^{2}}{2}\left[\omega_{0,1}, \omega_{0,1}\right]\right) d s \\
& =\pi_{1} k \int_{0}^{1} \varphi\left(\omega_{t} ; s\left(d t \wedge \alpha+\Omega_{t}\right)+\frac{s^{2}-s}{2}[\alpha, \alpha]\right) d s \text { on } P \times\{t\} \\
& =k(k-1) \int_{0}^{1} \int_{0}^{1} \varphi\left(\omega_{t}, s \alpha ; s \Omega_{t}+\frac{s^{2}-s}{2}[\alpha, \alpha]\right) d s d t .
\end{aligned}
$$

Using the formulas we have just derived and the interpretation of these forms, we are in a position to prove how the transgression form depends upon the connections.

Proof of Proposition (1.4). By (4.2), we have 


$$
\mu\left(\omega_{s+\Delta s}\right)-\mu\left(\omega_{s}\right)=\lambda\left(\omega_{s}, \omega_{s+\Delta s}\right) \varphi+d \mu\left(\omega_{s}, \omega_{s+\Delta s}\right) \varphi
$$

By definition, we know that

$$
\frac{\partial}{\partial s} T \varphi\left(\omega_{s}\right)=\frac{\partial}{\partial s} \mu\left(\omega_{s}\right) \varphi=\lim _{\Delta s \rightarrow 0} \frac{1}{\Delta s}\left\{\mu\left(\omega_{s+\Delta s}\right) \varphi-\mu\left(\omega_{s}\right) \varphi\right\}
$$

Therefore it suffices to prove the following.

LEMMA (4.1). If $\omega_{s}, s \in[0,1]$, is a smooth 1-parameter family of connections on $P$, then for $\varphi \in I^{k}(G)$, we have

$$
\lim _{\Delta s \rightarrow 0} \frac{1}{\Delta s} \lambda\left(\omega_{s}, \omega_{s+\Delta s}\right) \varphi=k \varphi\left(\frac{\partial}{\partial s} \omega_{s} ; \Omega_{s}\right) .
$$

Proof. By (4.4), $\lambda\left(\omega_{s}, \omega_{s+\Delta s}\right) \varphi=k \int_{0}^{1} \varphi\left(\omega_{s+\Delta s}-\omega_{s} ; \Omega_{s, t}\right) d t$ where $\Omega_{s, t}$ is the curvature form of the connection of the affine combination of $\omega_{s}$ and $\omega_{s+\Delta s}$, that is,

$\Omega_{s, t}=d\left(\omega_{s}+t\left(\omega_{s+\Delta s}-\omega_{s}\right)\right)+1 / 2\left[\omega_{s}+t\left(\omega_{s+\Delta s}-\omega_{s}\right), \omega_{s}+t\left(\omega_{s+\Delta s}-\omega_{s}\right)\right]$.

Letting $\Delta s$ approach zero, we have $\lim _{\Delta s \rightarrow 0} \Omega_{s, t}=d \omega_{s}+1 / 2\left[\omega_{s}, \omega_{s}\right]=$ $\Omega_{s}$. So we have

$$
\lim _{\Delta s \rightarrow 0} \frac{1}{\Delta s} \lambda\left(\omega_{s}, \omega_{s+\Delta s}\right) \varphi=k \int_{0}^{1} \varphi\left(\frac{\partial}{\partial s} \omega_{s} ; \Omega_{s}\right) d t=k \varphi\left(\frac{\partial}{\partial s} \omega_{s} ; \Omega_{s}\right) \text {. Q.E.D. }
$$

Similarly, if we use the Bott's formula (4.3), we can derive the formula given by Heitsch [4]. The following proof is given by Professor T. Nagano.

Proof of Corollary (2.2). By (4.3), we have

$$
\lambda\left(\omega_{s+\Delta s}, \omega\right) \varphi-\lambda\left(\omega_{s}, \omega\right) \varphi=-\lambda\left(\omega_{s}, \omega_{s+\Delta s}\right) \varphi+d \lambda\left(\omega_{s}, \omega_{s+\Delta s}, \omega\right) \varphi .
$$

But by Lémma (4.1), we see that

$$
\begin{aligned}
\frac{\partial}{\partial s} \lambda\left(\omega_{s}, \omega\right) \varphi & =\lim _{\Delta s \rightarrow 0} \frac{1}{\Delta s}\left\{\lambda\left(\omega_{s}+\Delta s, \omega\right) \varphi-\lambda\left(\omega_{s}, \omega\right) \varphi\right\} \\
& =-k \varphi\left((\partial / \partial s) \omega_{s} ; \Omega_{s}\right)+\text { exact form. }
\end{aligned}
$$

THEOREM 3. If $\omega_{s}, s \in[0,1]$, is a smooth 1-parameter family of flat connections, then for $\varphi \in I^{k}(G), k>1$,

$$
\left\{\mu\left(\omega_{0}\right) \varphi\right\}=\left\{\mu\left(\omega_{1}\right) \varphi\right\} \in H^{2 k-1}(P ; R) .
$$

Proof. Since $\omega_{0}, \omega_{1}$ are flat, $\mu\left(\omega_{0}\right) \varphi$ and $\mu\left(\omega_{1}\right) \varphi$ are closed forms on $P$. Put $\alpha=\omega_{1}-\omega_{0}, \bar{\omega}_{t}=\omega_{0}+t \alpha$ for $t \in[0,1], \bar{\Omega}_{t}=d \bar{\omega}_{t}+1 / 2\left[\bar{\omega}_{t}, \bar{\omega}_{t}\right]$.

By definition, we have $\lambda\left(\omega_{0}, \omega_{1}\right) \varphi=k \int_{0}^{1} \varphi\left(\alpha ; \bar{\Omega}_{t}\right) d t$. But by (2.1) and (2.2), we have $\bar{\Omega}_{t}=\left(t-t^{2}\right) D \alpha$. So $\lambda\left(\omega_{0}, \omega_{1}\right) \varphi=\left(k \int_{0}^{1}\left(t-t^{2}\right)^{k-1} d t\right) \varphi(\alpha ; D \alpha)$ 
$=c_{k} \varphi(\alpha ; D \alpha)$ where $c_{k}=k \int_{0}^{1}\left(t-t^{2}\right)^{k-1} d t=k !(k-1) ! /(2 k-1) !$.

By Proposition (3.3), $\{\varphi(\alpha ; D \alpha)\}=0$. Hence if we apply formula (4.2), it is clear to see that $\left\{\mu\left(\omega_{0}\right) \varphi\right\}=\left\{\mu\left(\omega_{1}\right) \varphi\right\}$. Q.E.D. It should be pointed out that Theorem 3 and Theorem 2 have the same contents.

Consider the example given in $\S 3, \mu\left(\omega_{0}\right) \varphi=2 \int_{0}^{1} \operatorname{tr}\left(\omega_{0} \wedge \Omega_{t}\right) d t$ where $\Omega_{t}=\left(t^{2}-t\right) \omega_{0} \wedge \omega_{0}$. By the fact that $\omega_{0} \wedge \omega_{0} \wedge \omega_{0}=6\left(\theta^{1} \wedge \theta^{2} \wedge \theta^{3}\right) I$, $\int_{0}^{1}\left(t^{2}-t\right) d t=-1 / 6$ we have $\mu\left(\omega_{0}\right) \varphi=-2 \theta^{1} \wedge \theta^{2} \wedge \theta^{3}$. Hence $\left\{\mu\left(\omega_{0}\right) \varphi\right\} \neq 0$ in $H^{3}(S O(3) ; R)$. It is easy to see that $\left\{\mu\left(\omega_{1}\right) \varphi\right\}=0$. By Theorem 3 , we have again obtained Proposition (3.5).

\section{BIBLIOGRAPHY}

1. R. Bott, Lecture on characteristic classes and foliation, Lecture Notes in Math., vol. 279, Springer-Verlag, Berlin and New York, 1972.

2. S. S. Chern, Geometry of characteristic classes, Proc. Thirteenth Biennial Seminar, Canadian Math. Soc., 1972, pp. 1-40.

3. S. S. Chern and J. Simons, Characteristic forms and geometric invariants, Ann. of Math. (2) 99 (1974), 48-69.

4. J. L. Heitsch, Deformations of secondary characteristic classes, Topology 12 (1973), 381-388. MR 47 \#9639.

5. S. Kobayashi and K. Nomizu, Foundations of differential geometry. Vols. I, II, Interscience, New York, 1963, 1969. MR 27 \#2945; 38 \#6501.

6. S. Kobayashi and T. Ochiai, G-structures of order two and transgression operators, J. Differential Geometry 6 (1971), 213-230. MR 46 \#823.

7. F. Kamber and P. Tondeur, Non-trivial characteristic invariants of homogeneous foliated bundles (preprint). 51-89.

8. Characteristic invariants of foliated bundles, Manuscripta Math. 11 (1974),

\section{DEPARTMENT OF MATHEMATICS, MICHIGAN STATE UNIVERSITY, EAST LANSING, MICHIGAN 48824}

Current address: Department of Mathematics, National Tsing Hua University, Taiwan, Republic of China 